\title{
Avoiding Ambiguity in Beamspace Processing
}

\author{
Ali Nasiri Amini, Student Member, IEEE, and Tryphon T. Georgiou, Fellow, IEEE
}

\begin{abstract}
In direction finding of narrow-band signals using antenna arrays with a large number of elements, the so-called beamspace matrix is often used to project the measurements into a lower dimension subspace. This reduces computation time and allows for parallel processing. On the other hand, beamspace preprocessing may introduce ambiguity, i.e., spurious estimated directions. We show that when the null space of the beamspace matrix is suitably designed, ambiguity within any sector of interest can be avoided.
\end{abstract}

Index Terms-Antenna arrays, direction of arrival (DOA) estimation, spectral analysis.

\section{INTRODUCTION}

$\mathbf{I}$ $\mathrm{N}$ direction of arrival (DOA) finding of narrow-band signals using array sensor measurements, the ambiguity arises when the array has identical responses to different sets of DOAs. A well-known example of ambiguity is in uniform linear array (ULA), when the intersensor spacing is greater than half of the wavelength of the impinging waves. We assume throughout that this is not the case; however, additional ambiguity may be introduced by beamspace preprocessing. This is the subject of this letter.

A number of high-resolution direction-finding techniques use beamspace preprocessing to reduce computation, improve performance in environments that include spatially colored noise, and enhance resolution. Among them, subspace direction-finding techniques such as MUSIC and ESPRIT are known as the most promising high-resolution algorithms. Combining beamspace preprocessing with subspace techniques has been suggested by a number of researchers (see [1, p. 1243] and references therein).

The essence of subspace techniques is eigendecomposition of sample spectral matrix (i.e., covariance matrix) to retrieve DOAs. The computational complexity of these algorithms is dominated by eigendecomposition that is of order $N^{3}$, with $N$ being the dimension of the spectral matrix. In order to reduce computation time and minimize numerical problems, the original data are projected into lower dimension subspace (i.e., the beamspace) before further processing. A well-designed projection matrix, i.e., beamspace matrix, may have additional advantages, such as attenuating strong interfering signals and whitening spatial color noise.

Manuscript received August 30, 2004; revised December 10, 2004. This work was supported in part by the National Science Foundation and the Air Force Office of Scientific Research. The associate editor coordinating the review of this manuscript and approving it for publication was Dr. Dimitri Van De Ville.

The authors are with the Department of Electrical and Computer Engineering, University of Minnesota, Minneapolis, MN 55455 USA (e-mail: nasiri@ece.umn.edu; tryphon@ece.umn.edu).

Digital Object Identifier 10.1109/LSP.2005.845596
Different schemes, such as conventional beams (Fourier beams), Dolph-Chebychev, and Taylor series, have been proposed for beamspace design (see [1, p. 1065]). In this letter, we show that applying such beamspace matrices often introduces spurious DOAs. To avoid such ambiguities, we propose a new scheme for designing the beamspace matrix.

The organization of the letter is as follows. In Section II, the subspace algorithms and beamspace versions of them are reviewed briefly. Then, the ambiguity problem of beamspace version of subspace methods is discussed. Subsequently, Section III gives two practical examples of ambiguity that are adopted from a recent textbook in array processing [1]. In Section IV, a specific class of beamspace matrices is presented whose members are shown to be free of ambiguities. Moreover, they have a nulling property that can be used to attenuate strong interfering signals. This is also verified by numerical examples at the end of this section. Concluding remarks are given in Section V.

\section{SubSPaCE Methods FOR DOA Estimation}

Consider a ULA that consists of $N$ sensors with intersensor spacing $d=\lambda / 2$. We refer to it as a standard linear array. Suppose $m$ uncorrelated plane waves impinge on it from different directions $\theta_{1}, \ldots, \theta_{m}$ with $m<N$. The following notation is used:

$$
\begin{aligned}
& \psi_{i}:=\cos \theta_{i} \\
& \Psi:=\left[\psi_{1}, \ldots, \psi_{m}\right] \\
& a(\psi):=\left[\begin{array}{llll}
1 & e^{j \pi \psi} & \ldots & e^{j(N-1) \pi \psi}
\end{array}\right]^{T} \\
& A(\Psi):=\left[\begin{array}{lll}
a\left(\psi_{1}\right) & \ldots & a\left(\psi_{m}\right)
\end{array}\right]
\end{aligned}
$$

where " $[\cdot]^{T}$ " denotes the transpose. The vector $a(\psi)$ is known as the steering vector, while $\Psi$ is the direction cosine vector. In the narrow-band snapshots model, the received snapshots are

$$
x(k)=A(\Psi) S(k)+v(k), \quad k=1,2, \ldots, K .
$$

Vectors $S(k)$ and $v(k)$ represent received signals and additive noise, respectively. Throughout, we assume that $\mathrm{E}\left\{v(k) v(k)^{*}\right\}=\sigma^{2} I$, where "[.]" denotes complex conjugate transpose, and $I$ is the identity matrix of the appropriate dimension. The direction-finding problem is to identify $\Psi$ from the snapshot record. Note that corresponding to each $\psi$, there are two directions $\theta$ and $-\theta$; however, this type of ambiguity is not the subject of this letter. We just consider the problem of retrieving $\Psi$ from the received snapshots.

A suitable eigendecomposition of the spectral matrix (covariance matrix) allows retrieval of $\Psi$. The spectral matrix is a second-order statistic of the snapshots, i.e.,

$$
T:=\mathrm{E}\left\{x(k) x(k)^{*}\right\} .
$$

This is usually estimated via

$$
\hat{T}=\frac{1}{K} \sum_{k=1}^{K} x(k) x(k)^{*} .
$$


Model (1) implies that

$$
\begin{aligned}
T & =A(\Psi) R A(\Psi)^{*}+\sigma^{2} I \\
R & :=\mathrm{E}\left\{S(k) S(k)^{*}\right\} .
\end{aligned}
$$

The matrix $R$ is diagonal since the impinging waves are assumed to be uncorrelated.

On the other hand, a singular value decomposition (SVD) of $T$ gives that

$$
T=U_{\mathrm{s}} \Lambda_{\mathrm{s}} U_{\mathrm{s}}^{*}+U_{n} \Lambda_{n} U_{n}{ }^{*}
$$

where $\left[\begin{array}{ll}U_{\mathrm{s}} & U_{n}\end{array}\right]$ is a unitary matrix, and the above summands correspond to splitting the contribution of the largest $m$ singular values from the rest. It is easy to show that

$$
\mathcal{R}(A(\Psi)) \equiv \mathcal{R}\left(U_{\mathrm{s}}\right)
$$

where " $\mathcal{R}$ " denotes the range of the matrix (i.e., column space). This range is referred to as the signal subspace, while its complement $\mathcal{R}\left(U_{n}\right)$ is referred to as the noise subspace (see [2, pp. 155-157]).

The signal subspace is fully specified by $\Psi$. Moreover, it can be shown that the correspondence

$$
\Psi \mapsto \mathcal{R}(A(\Psi))
$$

is one to one (see [2, pp. 155-157]). Therefore, $\Psi$ can be uniquely identified from the signal subspace, and there is no ambiguity. In practice, we work with the sample spectral matrix $\hat{T}$, and hence, the signal and noise subspaces can only be approximated. The variety in subspace algorithms (e.g., MUSIC, ESPRIT) stems from different estimation techniques for the signal subspace as well as different approximation schemes to fit a set of DOAs to the estimated subspace.

In the beamspace approach, a matrix $W^{n \times N}$ projects snapshots to a lower dimensional subspace. It is assumed that $N \gg$ $n>m$ and $W W^{*}=I$. Then, the eigenstructrue of matrix $T_{W}:=W T W^{*}$ is explored instead of $T$ to reduce the computational burden of subspace algorithms. In particular, SVD on $T_{W}$ gives

$$
T_{W}=U_{b s} \Lambda_{b s} U_{b s}^{*}+U_{b n} \Lambda_{b n} U_{b n}^{*}
$$

where $\left[\begin{array}{ll}U_{b s} & U_{b n}\end{array}\right]$ is again a unitary matrix, and the above summands correspond to splitting the contribution of the largest $m$ singular values from the rest. Equation (2) implies that

$$
T_{W}=W A(\Psi) R A(\Psi)^{*} W^{*}+\sigma^{2} I .
$$

It is easy to conclude from (6) and (7) that $\mathcal{R}(W A(\Psi)) \equiv$ $\mathcal{R}\left(U_{b s}\right)$. The similarity between this equation and (4) suggests that $\mathcal{R}\left(U_{b s}\right)$ may be utilized like a signal subspace in order to retrieve $\Psi$ and referred to as the beamspace signal subspace. An orthonormal basis for this subspace can be estimated via SVD of $W \hat{T} W^{*}$. Then, $\Psi$ is chosen to approximate $\mathcal{R}(W A(\Psi))$ with $\mathcal{R}\left(\hat{U}_{b s}\right)$ (see [1, pp. 1243-1251]). Different algorithms have been proposed to this end. For instance, similar to spectral MUSIC, DOAs are determined as the locations of the $m$ highest peaks of the function

$$
P(\psi)=\frac{1}{a^{*}(\psi) W^{*} \hat{U}_{b n} \hat{U}_{b n}^{*} W a(\psi)} .
$$

This function is known as "MUSIC pseudo-spectrum" (see [1, p. 1244]). Other variants of MUSIC and ESPRIT for beamspace are discussed in [3] and [4], respectively.

The pitfall in this methodology is that the counterpart of (5) does not hold for the beamspace signal subspace, i.e., the correspondence

$$
\Psi \mapsto \mathcal{R}(W A(\Psi))
$$

is not necessarily injective (i.e., one to one). In this case, there may be more than one $\Psi$ corresponding to $\mathcal{R}(W A(\Psi))$, which causes ambiguity.

It is easy to verify that the mapping (9) is an injection if and only if for any set of distinct $\left\{\psi_{1}, \psi_{2}, \ldots, \psi_{m+1}\right\}$, the vectors $\left\{W a\left(\psi_{1}\right), \ldots, W a\left(\psi_{m+1}\right)\right\}$ are linear independent. Thus, the maximum number of sources that can be identified without ambiguity can be assessed through the following definition.

Definition 1: Beamspace dimensionality of $W$, denoted by $d_{W}$, is the maximum integer $k$ such that if $\left\{\psi_{1}, \psi_{2}, \ldots, \psi_{k}\right\}$ are distinct, then the vectors $\left\{W a\left(\psi_{1}\right), \ldots, W a\left(\psi_{k}\right)\right\}$ are linear independent. ${ }^{1}$

Consequently, up to $m$ sources can be identified without ambiguity if and only if $m \leq\left(d_{W}-1\right)$.

\section{EXAMPLES OF AMBIGUITY}

One of the important applications of beamspace preprocessing is to modify subspace techniques for the cases that the additive noise is colored with unknown statistics. Beamspace matrices can be designed to act as a "spatial bandpass filter" that attenuates signals from sectors other than the sector of interest. By using parallel beamspace processors, $\psi$ range can be divided into small sectors. The white noise assumption is more realistic within these small sectors (see [1, p. 1063]). The following two examples that are adopted from [1] show that beamspace processors may produce spurious DOAs, even in the processing sector.

Example 1: We use the beamspace matrix proposed in Example 8.10.1 of [1, p. 1069]. Consider a standard ten-element linear array and the following matrix:

$$
B=\left[\begin{array}{ccccc}
1 & 1 & 1 & \ldots & 1 \\
0 & j & (2 j) & \ldots & (9 j) \\
0 & (j)^{2} & (2 j)^{2} & \ldots & (9 j)^{2}
\end{array}\right]
$$

which is a Taylor series matrix centered at $\psi=0$. The beamspace matrix $W$ is obtained via $W=\left(B B^{*}\right)^{-1 / 2} B$. It can be easily verified that $\{W a(-0.100), W a(-0.075), W a(0.338)\} \quad$ are linear dependent, which means that $d_{W} \leq 2$. Therefore, $\Psi_{1}=\{-0.100,-0.075\}, \Psi_{2}=\{-0.100,0.338\}$, $\Psi_{3}=\{-0.075,0.338\}$ produce identical beamspace signal subspaces. Consequently, a source or a jammer at $\psi=0.338$ may disguise itself as a source in the processing sector.

Example 2: Consider a standard 20-element linear array. We use a beamspace centered around $\psi=0$ that is obtained by orthogonalizing the following matrix:

$$
B=\left[\begin{array}{ccccc}
1 & e^{j(\pi / 25)} & e^{j(2 \pi / 25)} & \ldots & e^{j(19 \pi / 25)} \\
1 & 1 & 1 & \ldots & 1 \\
1 & e^{-j(\pi / 25)} & e^{j(2 \pi / 25)} & \cdots & e^{-j(19 \pi / 25)}
\end{array}\right] .
$$

${ }^{1}$ This is akin to dimensionality of the array manifold (see [5]). 

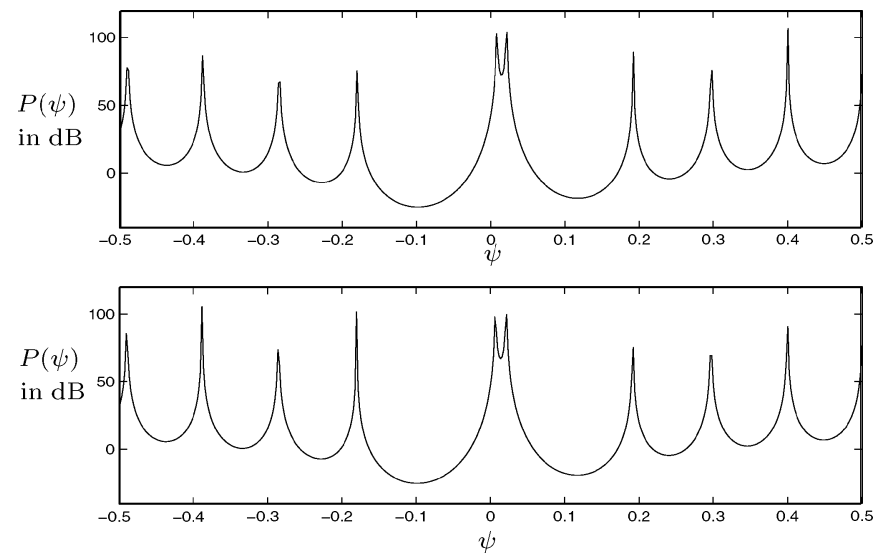

Fig. 1. MUSIC pseudo-spectrum. (Top) $\Psi_{1}=\{0.020,0.007\}$ (Bottom) $\Psi_{2}=\{0.020,-0.181\}$.

Such a beamspace matrix is referred to as the discrete Fourier transform (DFT) beamformer in the signal processing literature, and it is referred to as the Butler beamformer in the classic antenna literature. Putting the conventional beams closer than $2 \pi / N$ has been suggested in [1, p. 1066]. It can be verified that $\{W a(0.007), W a(0.020), W a(-0.181)\}$ are linear dependent. Therefore, applying this beamspace matrix when $m=2$ causes ambiguity.

Two scenarios are numerically explored where two equal-power (unit amplitude) uncorrelated signals are impinging on the array from $\Psi_{1}=\{0.020,0.007\}$ and $\Psi_{2}=\{0.020,-0.181\}$. The noise power is assumed $\sigma^{2}=0.01$, and the spectral matrix is estimated from 200 independent snapshots. Fig. 1 shows the MUSIC-pseudo-spectra for the two scenarios. It is seen that in both scenarios, there are many spurious peaks out of the processing sector. Note that in the second scenario, there exists spurious peak, even within the processing sector.

In practice, a well-designed $W$ provides high attenuation in sectors far from the processing sector; thus, sources or jammers located there do not disguise themselves. Yet, a certificate is needed to insure that sources within or close to the processing sector do not produce spurious DOAs in the sector of interest. Unfortunately, verifying such criterion for a given $W$ is not an easy task, since all possible DOA combinations in the vicinity of the processing sector should be checked. Yet, this is a case where design is easier than analysis.

\section{AMBIGUity-FreE BEAMSPACE DESIGN}

The main idea is to design $W$ such that the locations of the spurious DOAs are known and fixed, regardless of $\Psi$. Therefore, by removing these known spurious DOAs in the estimation process, ambiguity can be easily resolved. The drawback is that for these finite number of directions, ambiguity still exists. However, if these directions are selected outside of the sector of interest, then there is no ambiguity within that sector. To this end, the null space of $W^{n \times N}$, denoted by $\mathcal{N}(W)$, is specified such that it contains $N-n$ distinct evaluations of the steering vector at chosen directions. This can be explained in light of the following proposition.
Proposition 1: Suppose

$$
\mathcal{N}(W)=\operatorname{span}\left(a\left(\omega_{1}\right), \ldots, a\left(\omega_{N-n}\right)\right)
$$

and $\left\{\psi_{1}, \ldots, \psi_{\ell}\right\} \bigcap\left\{\omega_{1}, \ldots, \omega_{N-n}\right\}=\emptyset$. Then, for $\ell \leq n$, the matrix $W\left[a\left(\psi_{1}\right) \ldots a\left(\psi_{\ell}\right)\right]$ is full-column rank.

Proof: Note that $\left[a\left(\psi_{1}\right), \ldots, a\left(\psi_{\ell}\right)\right]$ has full-column rank. Thus, when $W\left[a\left(\psi_{1}\right), \ldots, a\left(\psi_{\ell}\right)\right]$ is column-rank deficient, there exist $\alpha_{i}$ 's such that

$$
\sum_{i=1}^{\ell} \alpha_{i} a\left(\psi_{i}\right) \in \mathcal{N}(W)
$$

This means

$$
\sum_{i=1}^{\ell} \alpha_{i} a\left(\psi_{i}\right)=\sum_{k=1}^{N-n} \beta_{k} a\left(\omega_{k}\right)
$$

and then, $\left[a\left(\omega_{1}\right), \ldots, a\left(\omega_{N-n}\right), a\left(\psi_{1}\right), \ldots, a\left(\psi_{\ell}\right)\right]$ are linearly dependent. Hence, since any distinct set of $\left\{a\left(\psi_{1}\right) \ldots a\left(\psi_{N}\right)\right\}$ are linear independent, we conclude that if $\ell \leq n$, then $W\left[a\left(\psi_{1}\right) \ldots a\left(\psi_{\ell}\right)\right]$ is full-column rank.

Thus, with (10) in place and $\psi \notin\left\{\omega_{1}, \ldots, \omega_{N-n}\right\}$, $W a(\psi) \in \mathcal{R}\left(U_{b s}\right)$ implies that $\psi$ is an actual source direction (i.e., $\psi \in \Psi$ ). In other words, the only possible spurious DOAs are $\left\{\omega_{1}, \ldots, \omega_{N-n}\right\}$, which we have already specified at innocuous locations. One such choice for the beamspace matrix is the well-known conventional beams, where $n$ rows of the DFT matrix are chosen to form $W$ (see [1, p. 1066]). In this case, the removed rows specify $N-n$ null directions.

In radar and communication applications where the location of the jamming signals is roughly available, it is natural to attenuate the spatial sector around them. This calls for a beamspace design scheme that provides attenuation in this particular spatial sector (i.e., the interval $E=\left[\psi_{l}, \psi_{u}\right]$ ) as well as a certificate for nonambiguous DOA estimation in the complement of $E$ (i.e., $\left.E^{c}\right)$. We present such a design scheme in the sequel.

If columns of $C$ form an orthonormal basis for $\mathcal{N}(W)$, then $\mathrm{W}$ is effectively a completion of $C$ into a unitary matrix [C $\left.W^{*}\right]$. Thus, equivalently, $C$ can be designed instead of $W$. To ensure attenuation within the interval $E=\left[\psi_{l}, \psi_{u}\right]$, it is natural to minimize a gain functional such as

$$
\int_{E}\|W a(\psi)\|^{2} d \psi
$$

This requirement is equivalent ${ }^{2}$ to maximization of

$$
\begin{aligned}
\int_{E}\left\|C^{*} a(\psi)\right\|^{2} d \psi & =\operatorname{trace}\left(C^{*} \int_{E} a(\psi) a(\psi)^{*} d \psi C\right) \\
& =\sum_{i=1}^{N-n} c_{i}^{*} D c_{i}
\end{aligned}
$$

where $c_{i}$ 's are columns of $C$, and elements of the square matrix $D$ are

$$
d_{p q}= \begin{cases}\frac{\left(e^{j \pi(p-q) \psi_{u}}-e^{j \pi(p-q) \psi_{l}}\right)}{j \pi(p-q)} & p \neq q ; \\ \left(\psi_{u}-\psi_{l}\right) & p=q .\end{cases}
$$

${ }^{2}$ To see this, note that

$$
\begin{aligned}
\int_{E}\|W a(\psi)\|^{2} d \psi+\int_{E}\left\|C^{*} a(\psi)\right\|^{2} d \psi & = \\
\int_{E}\left\|\left[W C^{*}\right] a(\psi)\right\|^{2} d \psi & =N\left(\psi_{u}-\psi_{l}\right) .
\end{aligned}
$$




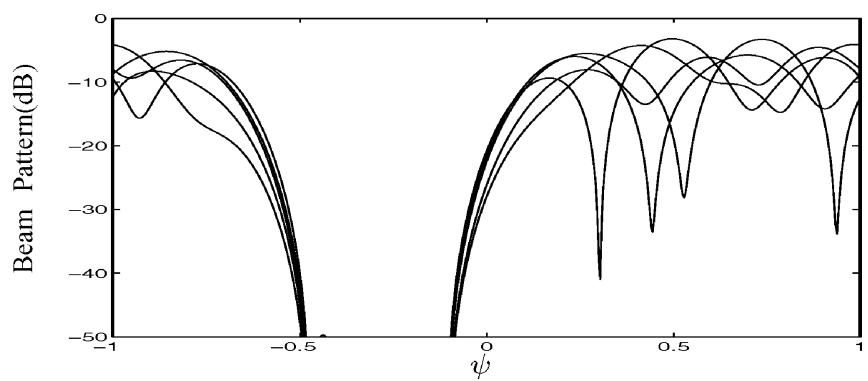

Fig. 2. Beam patterns for $W_{1}$.

This optimization problem can be interpreted alternatively as maximizing the gain functional (11) over the interval $E^{c}$ (e.g., the processing sector). ${ }^{3}$ The reason is that the integral of the gain functional (11) over $[-1,1]$ is constant, i.e.,

$$
\int_{E}\|W a(\psi)\|^{2} d \psi+\int_{E^{c}}\|W a(\psi)\|^{2} d \psi=2 n .
$$

To avoid ambiguity within the processing sector (i.e., $E^{c}$ ), $W$ is subject to have $N-n$ null directions in $E$. Note that maximizing

$$
\sum_{i=1}^{N-n} c_{i}^{*} D c_{i}
$$

subject to the existence of $N-n$ directions in $E$ such that $C\left[a\left(\omega_{1}\right) \ldots a\left(\omega_{N-n}\right)\right]=0$ is not a convex optimization problem. A simple and yet effective heuristic algorithm that successively chooses a null direction that maximizes the corresponding summand in (12) is given below.

1) $\quad a_{0}(\omega)=a(\omega) /\|a(\omega)\|, c_{0}=0$

2) for $k=1:(N-n)$

a)

$$
a_{k}(\omega)=\frac{\left(I-c_{k-1} c_{k-1}^{*}\right) a_{k-1}(\omega)}{\left\|\left(I-c_{k-1} c_{k-1}^{*}\right) a_{k-1}(\omega)\right\|}
$$

b) $\quad \omega_{k}=\arg \max _{\psi_{l} \leq \omega \leq \psi_{u}} a_{k}(\omega)^{*} D a_{k}(\omega)$

c) $c_{k}=a_{k}\left(\omega_{k}\right)$.

The simulation below highlights the potential of this technique for the beamspace design.

Example 3: Consider a standard ULA with $N=10$. Two equal-power uncorrelated signals impinge from directions $\psi_{1}=0.51$ and $\psi_{2}=0.55$. Also, three jammers with power $10 \mathrm{~dB}$ less than signals impinge from the band $-0.6<\psi<0$ that are simulated by a uniform distribution in this band. We use $K=200$ independent snapshots and project them by two beamspace matrices with $n=5$. The first beamspace matrix $W_{1}$ is computed by the proposed algorithm when $\psi_{l}=-0.6, \psi_{u}=0$. The null directions are computed as $[-0.1091,-0.1939,-0.2970,-0.3818,-0.4727]$, and its beam patterns are plotted in Fig. 2. The second beamspace matrix $W_{2}$ is the conventional beam matrix with five beams around the midpoint $\psi=(0.51+0.55) / 2$.

${ }^{3}$ This is akin to discrete prolate spheroidal sequences (DPSS) beamspace (see $[1$, p. 1069]). The difference is that here, $W$ is subject to have $N-n$ null directions outside the processing sector.
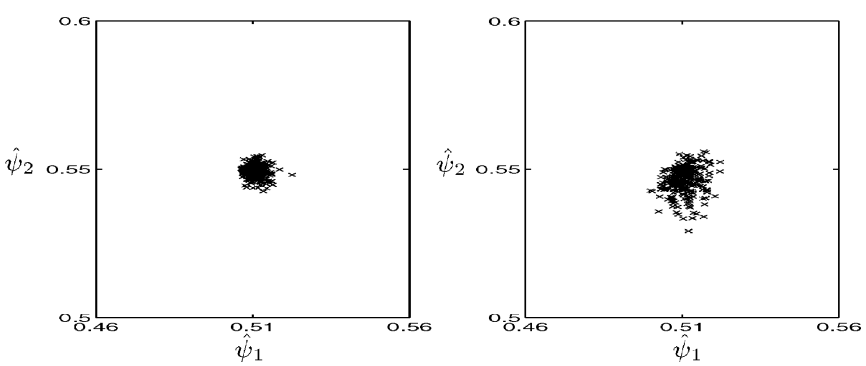

Fig. 3. Scatter diagram of the estimated DOAs. (Left) Applying $W_{1}$ (proposed beamspace). (Right) Applying $W_{2}$ (conventional beams).

TABLE I

RESULTS OF SIMULATION IN EXAMPLE 3

\begin{tabular}{c|c|c|c|c|c|c}
\hline \multirow{2}{*}{$\psi$} & \multicolumn{3}{|c|}{$\mathrm{W}_{1}$} & \multicolumn{3}{c}{$\mathrm{W}_{2}$} \\
\cline { 2 - 7 } & Bias & Var & Fail. rate & Bias & Var & Fail. rate \\
\hline 0.51 & $0.9 \mathrm{e}-3$ & $4.9 \mathrm{e}-6$ & 0.00 & $0.8 \mathrm{e}-3$ & $1.3 \mathrm{e}-5$ & 0.14 \\
0.55 & $0.6 \mathrm{e}-3$ & $3.6 \mathrm{e}-6$ & 0.00 & $3.9 \mathrm{e}-3$ & $1.9 \mathrm{e}-5$ & 0.14 \\
\hline
\end{tabular}

We use the spectral MUSIC algorithm to identify sources. Fig. 3 is the scatter diagram of the estimated $\psi_{1}$ and $\psi_{2}$ resulted from 300 simulations. It is seen that $W_{1}$ generates better estimates for DOAs. Table I contains the statistical properties of the identified DOAs as well as failure rates. A failure occurs when the MUSIC pseudo-spectrum does not have two peaks in the sector of interest. It is clear that $W_{1}$ outperforms $W_{2}$.

\section{CONCLUDing REMARKS}

We presented examples of well-known beamspace processors that produce spurious DOAs. It was shown that beamspace may introduce spurious DOAs unless the number of sources is less than the beamspace dimensionality. Thus, for a reliable direction-finding algorithm, the beamspace dimensionality should be determined. On the other hand, calculating the beamspace dimensionality requires an exhaustive search over all possible direction combinations, which is impossible in practice. We presented a specific class of beamspace matrices that can be applied as preprocessors without the risk of ambiguity. It is shown that this class is rich enough to accommodate practical demands that motivate beamspace processing.

\section{ACKNOWLEDGMENT}

The authors would like to thank Prof. M. Kaveh, M. S. Takyar, and anonymous reviewers for their valuable comments and suggestions.

\section{REFERENCES}

[1] H. L. Van Trees, Optimum Array Processing. New York: Wiley, 2002.

[2] P. Stoica and R. Moses, Introduction to Spectral Analysis. Upper Saddle River, NJ: Prentice-Hall, 1997.

[3] M. D. Zoltowski, G. M. Kautz, and S. D. Silverstein, "Beamspace rootMUSIC," IEEE Trans. Signal Proces., vol. 41, no. 1, pp. 344-364, Jan. 1993.

[4] G. Xu, S. D. Silverstein, R. Roy, and T. Kailath, "Beamspace ESPRIT,' IEEE Trans. Signal Process., vol. 42, no. 2, pp. 349-356, Feb. 1994.

[5] C. Proukakis and A. Manikas, "Study of ambiguities of linear arrays," in Proc. IEEE Int. Conf. Acoust., Speech, Signal Process., vol. IV, 1994, pp. 549-552. 